\title{
A "Sidewinding" Locomotion Gait for Hyper-Redundant Robots
}

\author{
J.W. Burdick, J. Radford \\ Dept. of Mechanical Engineering, Mail Code 104-44, CALTECH, Pasadena, CA 91125.
}

G.S. Chirikjian

Dept. of Mechanical Engineering, 124 Latrobe Hall, Johns Hopkins University, Baltimore, MD 21218.

\begin{abstract}
This paper considers the kinematics of a novel form of hyper-redundant mobile robot locomotion which is analogous to the 'sidewinding' locomotion of desert snakes. This form of locomotion can be generated by a repetitive traveling wave of mechanism bending. Using a continuous backbone curve model, we develop algorithms which enable travel in a uniform direction as well as changes in direction.
\end{abstract}

\section{Introduction}

Hyper-redundant robotic systems have a very large or infinite degree of kinematic redundancy. They are analogous to snakes, worms, or elephant trunks. In previous work [ChB91a, Ch92], two of the authors have developed novel methods for kinematic analysis and modeling of such systems. Further, these basic methods were subsequently used to develop efficient algorithms for hyperredundant robot obstacle avoidance [ChB90a], locomotion [ChB91b, ChB93a, ChB93b], and grasping [ChB91b, Ch92, ChB93b].

We define hyper-redundant robot "locomotion" as the net displacement of a hyper-redundant mobile robot which arises from internally induced bending and twisting of the mechanism. Actuatable wheels, tracks, or legs are not necessary. A gait is a distinct repetitive sequence of mechanism deformations which results in net displacement. In [ChB91b, ChB93a, ChB93b] we developed two classes of hyper-redundant robot locomotion gaits which are based on standing or traveling waves of mechanism distortion. These gaits are idealizations of gaits used by inchworms, earthworms, and slugs. These locomotion schemes have been implemented, and their viability demonstrated in an actual 30 degree-of-freedom hyper-redundant robot mechanism [ChB92b, Ch92, ChB93a]. However, other possible forms of hyper-redundant locomotion do not fit into these gait classes. Snakes also employ sidewinding, concertina, and undulating gaits [Gray]. This paper develops a novel locomotion scheme which is qualitatively identical to sidewinding. This gait is a useful addition to our previously developed gait repetoire. Empirical evidence indicates that snakes generate larger accelerations and travel faster when employing a sidewinding gait. Thus, sidewinding would be most useful for implementing fast gross displacement, while the algorithms in [ChB93a, ChB93b] would be most useful for precise locomotory movements.
While some mobile hyper-redundant robots are a hybrid between a snake-like vehicle and a tracked or wheeled vehicle [HiMo90], the focus of this paper and its companions [ChB91b, ChB93a, ChB93b] is lgcomotion which arises from internal robot hyper-redundant robotic locomotion research can be found in [ChB93a]. While the robotics literature is devoid of sidewinding gait analysis, biologists have empirically examined sidewinding [Gray, Secor, SJB]. Here we give the first quantitative description of a sidewinding motion.

\section{Qualitative Description of Sidewinding}

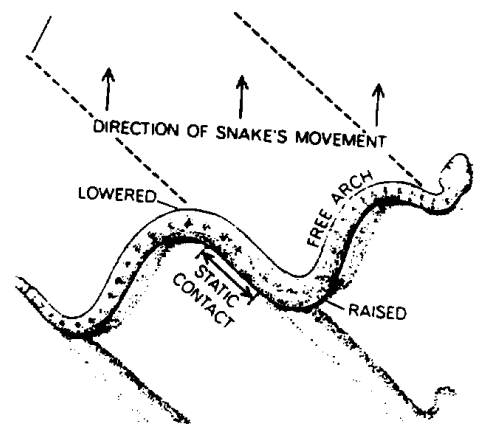

Figure 1: Sidewinding snake

If the snake is moving uniformly across a sandy surface, a set of parallel tracks, which are neither parallel nor perpendicular to the direction of motion, will be left in the sand after the snake passes (Fig. 1). Let these tracks be called ground contact tracks, or GCTs. The portions of the snake in contact with the ground will henceforth be termed ground contact segments, or GCSs. Net snake displacement, or locomotion, is produced by moving the snake to sequential GCTs.

The cycle begins with the head of the snake lifting from a current GCT and moving toward the next GCT. The lifted portion is termed an arch segment, or AS. The body is peeled away from the most forward GCT until almost 1/4 of the snake's body is cantilevered. A point just behind the head touches the ground, establishing a point on the next GCT. Successive body segments are "layed" down on along this newly established GCT, while segments are simultaneously "peeled" away from the prior GCT. In this way, a GCS effectively travels the length of a GCT, even though the GCSs are at rest with respect to the ground. 
After a certain amount of the snake is in contact most snakes, the body straddles three GCTs, or two GCTs during transitory phases. The combination of an adjacent GCS and an AS will be termed a basic gait segment. The net direction of travel is the sum of two components: one parallel to the GCT and one along a vector from the beginning of one AS to the beginning of the next.

Experiments indicate that sidewinding generates the greatest acceleration and speed of all gaits. This is logical, as: (1) the GCSs afford a large surface area of contact between the ground and the snake; (2) the contact friction is static, and not sliding, friction; and (3) the contact reaction forces are distributed over two or more GCSs, adding stability. Additionally, desert snakes prefer sidewinding because only small portions of the snake are in contact with hot desert sand, thus preventing overheating.

\section{Kinematics of Backbone Curves}

In the rest of this paper, we abstract the important macroscopic kinematic phenomena in terms of a continuous backbone curve. In this paradigm, which is the basis of our previous work, motion planning is reduced to determining the proper time varying behavior of the backbone curve. We review here the essential kinematics of nonextensible backbone curves.

The Cartesian position of points on a nonextensible backbone curve can be intrinsicly parametrized by:

$$
\vec{x}(s, t)=\int_{0}^{s} \vec{u}(\sigma, t) d \sigma
$$

where $s$ is the arclength parameter at time $t$ : i.e., $|d \vec{x} / d s|=1 . \vec{x}(s, t)$ is a vector from the base of the backbone curve, located at $s=0$, to the point on the backbone curve denoted by $s$. Distance is normalized so that $s \in[0,1] . \vec{u}(s, t)$ is the unit length backbone curve tangent at $s$. The parametrization of (3.1) has the following interpretation. The backbone curve is "grown" from the base by propagating the curve forward along the tangent vector, which is varying its direction according to $\vec{u}(s, t)$. Any spherical kinematic representation can be used to parametrize $\vec{u}(s)$ in (3.1) [ChB92c]. Here we use the following parametrization for $\vec{x}(s, t)$ :

$$
\left[\begin{array}{l}
x_{1}(s, t) \\
x_{2}(s, t) \\
x_{3}(s, t)
\end{array}\right]=\left[\begin{array}{c}
\int_{0}^{s} \sin K(\sigma, t) \cos T(\sigma, t) d \sigma \\
\int_{0}^{s} \cos K(\sigma, t) \cos T(\sigma, t) d \sigma \\
\int_{0}^{s} \sin T(\sigma, t) d \sigma
\end{array}\right] .
$$

$K(s)$ and $T(s)$ are angles which determine the direction of $\vec{u}(s)$ (see Fig. 2). By convention, the initial conditions $K(0)=T(0)=0$ are assumed. In summary, the backbone curve is a function of a reduced set of intrinsic "shape functions," $K(s, t)$ and $T(s, t)$. Additionally, the roll distribution

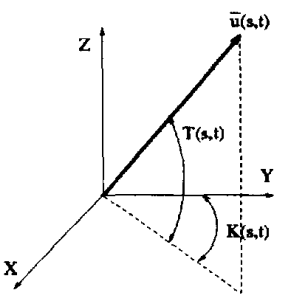

Figure 2: Definitions of $K(s, t), T(s, t)$

[ChB91a], which measures how the robot twists about the backbone, is required to completely specify a hyper-redundant robot configuration. Assuming an axially symmetric robot, roll can be neglected. The backbone curve is an abstraction of a real hyper-redundant robot geometry. To apply this framework to discretely segmented hyperredundant morphologies, a "fitting" procedure is required. Fitting procedures determine the actuator displacements so that the discrete morphology robot exactly or closely follows the backbone curve model [ChB91a, Ch92]. The spatial Stewart platform fitting algorithm of [ChB91a] is used in the ensuing example.

\section{Uniform Direction Sidewinding}

Here we consider uniform motion oyer flat terrain. winding can viewed as a form of traveling wave locomotion, in which the AS shape propagates from the head to the tail. For uniform motion, the AS wave shape is constant. For turning motions, the AS shape is time varying. This section synthesizes the backbone curve shape functions which implement a uniform direction sidewinding gait over flat terrain. This can be done as follows: (1) find the static AS and GCS shapes; (2) ensure that the AS and GCS shapes blend smoothly at their intersection; and (3) convert the resulting shape to a traveling wave form (i.e., for shape function $\mathcal{S}(s, t)$ : $\mathcal{S}(s, t) \rightarrow \mathcal{S}(s-\omega t, t))$. The backbone curve shape can in turn be used to control a discrete morphology system through a fitting procedure.

We assume that $s=0$ is the "head" of the packbone curve, while $s=1$ is the "rear" of the backbone curye. Also assume the robot contacts the
ground via $N$ GCSs. Similarly, there will be $N$ basic gait segments (some of these basic gaits segments may be not be fully formed at any instant). Index these segments by $j$, with $j=1$ indexing the segment closed to the head. Let the arc-length of the $j^{\text {th }}$ fully formed GCS be denoted by $L_{g_{j}}$. Similarly, let $L_{a_{j}}$ be the arc-length of a fully formed AS. Let $s_{j 1}(t)$ and $s_{j 2}(t)$ denote the most forward and rearward points of the $j^{\text {th }}$ GCS (see Fig. 3). Similarly, let $s_{j 3}(t)$ denote the most rearward point of the $j^{\text {th }}$ AS. $s_{j 1}(t), s_{j 2}(t)$, and $s_{j 3}(t)$ move along the backbone curve with wave speed $\omega$.

The total backbone shape can then be constructed as the piecewise sum of shape functions which sep- 


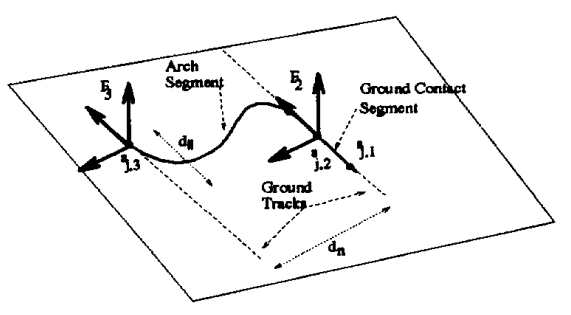

Figure 3: Schematic of an arch segment

arately control the shapes of the arch segments and the ground contact segments:

$$
\begin{aligned}
& K(s, t)=\sum_{j=1}^{N} K_{g}\left(s-\alpha_{t_{j}}(t)\right) W\left(s-\alpha_{t_{j}}(t), s_{j, 1}, s_{j, 2}\right) \\
& +\quad K_{a}\left(s-\alpha_{t_{j}}(t)\right) W\left(s-\alpha_{t_{j}}(t), s_{j, 2}, s_{j, 3}\right) \\
& T(s, t)=\sum_{j=1}^{N} T_{g}\left(s-\alpha_{t_{j}}(t)\right) W\left(s-\alpha_{t_{j}}(t), s_{j, 1}, s_{j, 2}\right) \\
& +\quad T_{a}\left(s-\alpha_{t_{j}}(t)\right) W\left(s-\alpha_{t_{j}}(t), s_{j, 2}, s_{j, 3}\right)
\end{aligned}
$$

where $K_{a}(s, t), T_{a}(s, t), K_{g}(s, t)$, and $T_{g}(s, t)$ are respectively the static shape functions for the arch and ground contact segments of the backbone curve. $\alpha_{t_{j}}(t)$ takes the form [ChB91b]:

$$
\alpha_{t_{j}}(t)=\omega\left[t-I\left(t, \frac{L_{a_{j}}+L_{g_{j}}}{\omega}\right)\right]-L_{a_{j}}
$$

where $I\left(t, t_{p}\right)=t_{p}$ (int) $\left(t / t_{p}\right)$ and (int) is the greatest integer function. This choice of $\alpha_{t}(t)$ induces a constant velocity bending wave, with speed $\omega$, to travel the length of the backbone curve. The wave repeats at period $\left(L_{a}+L_{g}\right) / \omega$, causing continuous periodic motion. $W(\cdot, \cdot, \cdot)$ is a "window function:

$$
W\left(s, s_{0}, s_{1}\right)= \begin{cases}1 & \text { for } s \in\left[s_{0}, s_{1}\right] \\ 0 & \text { otherwise }\end{cases}
$$

The GCSs are straight lines for uniform sidewinding motion over flat ground. Consequently, $K_{g}(s, t)$ and $T_{g}(s, t)$ assume the simple forms:

$$
K_{g}(s, t)=0 ; \quad T_{g}(s, t)=0
$$

The next subsection develops constraints for the arch segment shape functions.

\subsection{The Arch Segment Shape Functions}

Assign two local coordinate systems, $F_{2}$ and $F_{3}$, at $s_{j, 2}$ and $s_{j, 3}$ (Fig. 3 ). Let the $x$-axes of these coordinate frames, denoted $\hat{\mathbf{x}}_{j, 2}$ and $\hat{\mathbf{x}}_{j, 3}$, be collinear with the GCTs. The respective $z$-axes are normal to the plane of locomotion. Let $\xi=\left(s-s_{j, 2}\right) / L_{a_{j}}$, i.e. $\xi \in[0,1]$, be a normalized distance on the AS: Points along the AS, as measured in $F_{0}$, can be parametrized as:

$$
\vec{p}(\xi)=\left(\begin{array}{l}
p_{1}(\xi) \\
p_{2}(\xi) \\
p_{3}(\xi)
\end{array}\right)=L_{a_{j}} \int_{0}^{\xi} \vec{u}_{a}(\sigma) d \sigma
$$

where $\vec{u}_{a}(s)$ is the vector tangent to the AS in the interval $s \in\left[s_{j, 2}, s_{j, 3}\right] . \vec{u}_{a}$ is parameterized by $K_{a}$ and $T_{a}$, as in Equation (3.2). In this local coordinate system, the arch segment must satisfy:

$$
\begin{aligned}
\vec{p}(0) & =\hat{\mathbf{x}}_{j, 2}=\text { tangent to GCT at } s_{j, 2} \\
\vec{p}(1) & =\vec{x}\left(s_{j, 3}\right)-\vec{x}\left(s_{j, 2}\right)=\left[d_{\|}, d_{n}, 0\right]^{T} \\
\vec{p}(1) & =\hat{\mathbf{x}}_{j, 3}=\text { tangent to GCT at } s_{j, 3}
\end{aligned}
$$

where a 'indicates differentiation with respect to s. $d_{n}$ is the distance between the parallel ground contact tracks, while $d_{\|}$is the displacement of two ends of the arch segment along the ground contact line direction (Fig. 3 ).

For the case of parallel ground contact lines employed in uniform motion, $\hat{\mathbf{x}}_{j, 2}=\hat{\mathbf{x}}_{j, 3}=[1,0,0]^{T}$. Thus, to satisfy condition (4.7), we require that:

$$
\begin{gathered}
K_{a}\left(s_{j, 2}\right)=K_{a}\left(s_{j, 3}\right)=T_{a}\left(s_{j, 2}\right)=T_{a}\left(s_{j, 3}\right)=0 \\
L_{a} \int_{0}^{1} \cos K_{a}(\xi) \cos T_{a}(\xi) d \xi=d_{\|} \\
L_{a} \int_{0}^{1} \sin K_{a}(\xi) \cos T_{a}(\xi) d \xi=d_{n} \\
L_{a} \int_{0}^{1} \sin T_{a}(\xi) d \xi=0 \\
\int_{0}^{\xi} \sin T(\xi) d \xi \geq 0 \forall \xi \in[0,1]
\end{gathered}
$$

where (4.10) guarantees that the AS does not touch the ground between its end points. Any $K_{a}(s)$, $T_{a}(s)$ which satisfy Equations (4.8), (4.9), and (4.10) are suitable candidates for the arch shape functions.

The problem of finding the AS shape functions can also be posed as a hyper-redundant robot inverse kinematic problem. The $j^{\text {th }}$ AS is akin to a hyperredundant manipulator with base frame aligned with $F_{2}$ at $\vec{x}\left(s_{j, 2}\right)$, and whose tip must reach $\vec{x}\left(s_{j, 3}\right)$ with constraints on orientation. Schepnes for solvSection 3 can be found in [ChB91a,ChB92a, Ch92]. In [ChB92a], the calculus of variations is used to find the shape functions which satisfy boundary conditions while also minimizing a criteria, such as total robot bending. This approach is physically appealing, as it is likely that real snakes choose the AS shape to minimize some criteria. However, this approach is computationally less attractive for practical applications. Alternatively, a 
'modal' approach can be used to efficiently compute inverse kinematic solutions in a practically useful way [ChB91a, ChB92c]. In this approach, the shape functions are restricted to a "modal" form:

$$
\begin{aligned}
K_{a}(s, t) & =\sum_{i=1}^{N_{K}} a_{i}(t) \phi_{i}(s) \\
T_{a}(s, t) & =\sum_{i=N_{K}+1}^{N_{K}+N_{T}} a_{i}(t) \phi_{i}(s) .
\end{aligned}
$$

where the $\left\{\phi_{i}(s)\right\}$ are mode functions, while the $\left\{a_{i}(t)\right\}$ are modal participation factors. $N_{K}$ and $N_{T}$ are respectively the number of modes distributed in the $K$ and $T$ shape functions. Their number must equal or exceed the number of geometric constraints. The $\left\{\phi_{i}\right\}$ are specified by the user. For given $\left\{\phi_{i}\right\}$, the AS shape is determined by the $\left\{a_{i}\right\}$. Inverse kinematics, reduces to the search for the proper $\left\{a_{i}\right\}$ which satisfy the boundary conditions. The next section shows by example how the mode shapes can be chosen to reflect the physical characteristics of the problem. For more details of this approach, see the appropriate references.

\subsection{An Example}

For uniform motion on flat ground, $F_{3}$ is displaced relative to $F_{2}$ with only two degrees of freedom. Thus $K_{a}$ and $T_{a}$ can assume the form:

$$
K_{a}(s, t)=a_{1}(t) \phi_{1}(s) ; \quad T_{a}(s, t)=a_{2}(t) \phi_{2}(s)
$$

To satisfy $(4.8), \phi_{1}(s)$ and $\phi_{2}(s)$ must be zero at $s_{j, 2}$ and $s_{j, 3}$. Additional physical insight into reasonable choices for $\phi_{1}(s)$ and $\phi_{2}(s)$ can be realized as follows. $\sin T_{a}(s)$ encodes the vertical component of $\vec{u}_{a}$. While $T_{a}(s)$ need not have any symmetry, for simplicity's sake we assume $\phi_{2}(s)$ is odd about the midpoint of the interval $\left[s_{j, 2}, s_{j, 3}\right]$. Thus, the maximum height of the arch segment occurs at $\xi=\frac{1}{2} . K_{a}$ encodes the angle between the projection of $\vec{u}_{a}(s)$ onto the locomotion plane and the $x_{2}$-axis. Though $K_{a}(s)$ need not have any symmetry, we again assume that $K_{a}(s)$ is even about the midpoint of the arch segment. In this way, the arch segment has a nominally "S-shaped" geometry. A simple set of shape functions which satisfies the above constraints is:

$$
\begin{gathered}
\phi_{1}(\xi)= \begin{cases}2 \pi \xi & \text { for } \xi \in\left[0, \frac{1}{2}\right] \\
2 \pi(1-\xi) & \text { for } \xi \in\left[\frac{1}{2}, 1\right]\end{cases} \\
\phi_{2}(\xi)= \begin{cases}2 \pi \xi & \text { for } \xi \in\left[0, \frac{1}{4}\right] \\
\pi(1-2 \xi) & \text { for } \xi \in\left[\frac{1}{4}, \frac{3}{4}\right] \\
2 \pi(\xi-1) & \text { for } \xi \in\left[\frac{3}{4}, 1\right]\end{cases}
\end{gathered}
$$

Substituting (4.13) and (4.14) into (3.2), a tedious calculation shows that at $\xi=1$ :

$$
\begin{aligned}
p_{1}(1) & =x_{1}\left(s_{j, 3}\right)-x_{1}\left(s_{j, 2}\right) \\
& =\frac{2 \cos \left(\frac{\pi a_{1}}{2}\right)}{\pi\left(a_{1}^{2}-a_{2}^{2}\right)}\left[a_{1} \sin \left(\frac{\pi a_{1}}{2}\right)-a_{2} \sin \left(\frac{\pi a_{2}}{2}\right)\right] L_{a} \\
p_{2}(1) & =x_{2}\left(s_{j, 3}\right)-x_{2}\left(s_{j, 2}\right) \\
& =\frac{2 \sin \left(\frac{\pi a_{1}}{2}\right)}{\pi\left(a_{1}^{2}-a_{2}^{2}\right)}\left[a_{1} \sin \left(\frac{\pi a_{1}}{2}\right)-a_{2} \sin \left(\frac{\pi a_{2}}{2}\right)\right] L_{a} \\
p_{3}(1) & =x_{3}\left(s_{j, 3}\right)-x_{3}\left(s_{j, 2}\right)=0
\end{aligned}
$$

Dividing dividing the $x_{2}$ component by the $x_{1}$ component, it can be seen that:

$$
a_{1}=\frac{2}{\pi} \operatorname{Atan} 2\left[d_{n}, d_{\| l}\right] .
$$

Squaring and adding the first two components of (4.15), $a_{2}$ can be found as the root to the following transcendental equation:

$$
\beta a_{2}^{2}-a_{2} \sin \left(\frac{\pi a_{2}}{2}\right)=\beta a_{1}^{2}-a_{1} \sin \left(\frac{\pi a_{1}}{2}\right)
$$

where $a_{1}$ assumes the value computed using Equation (4.16). This has two symmetric roots which lead to the same shape.

These simple mode functions are advantageous because they lead to nearly closed form solutions for the $\left\{a_{i}\right\}$. However, there are infinitely many other modal expansions of $K_{a}$ and $T_{a}$ which satisfy constraints (4.8), (4.9), and (4.10), but do not have closed form solutions as above. The choice of particular mode functions is often not highly critical to the success of this method. The modal participation factors can be efficiently computed in these cases by using a method analogous to the "resolved rate" trajectory planning approach. This method is based on "modal Jacobian" [ChB92b].

Fig. 4 shows the concatenation of the ASs and GCSs. This system will sidewind if the backbone curve is given the traveling wave form of Equations (4.1) and (4.2). Fig. 5 shows the ground contact tracks left by the locomotion of this sidewinding example, while Fig. 6 shows a sequence from a computer simulation of the sidewinding gait described in this section. If we define the "average velocity," $\langle v\rangle$, of the sidewinding gait to be the net displacement that the tail of the backbone curve makes during one complete wave cycle, then it can be shown that:

$$
<v>=\frac{N \sqrt{\left(L_{a}-d_{\|}\right)^{2}+d_{n}^{2}}}{\omega}
$$

Note that sidewinding gaits can also be implemented in robotic mechanisms which do not have a continuous morphology. Fig. 7 shows snapshots from a computer simulation of hyper-redundant 


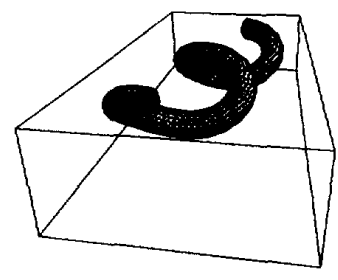

Figure 4: Idealized Sidewinding Shape

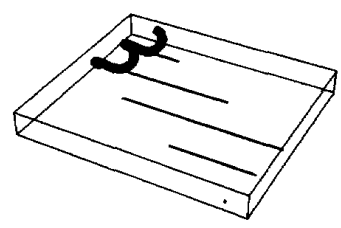

Figure 5: Geometry of ground tracks for sidewinding example
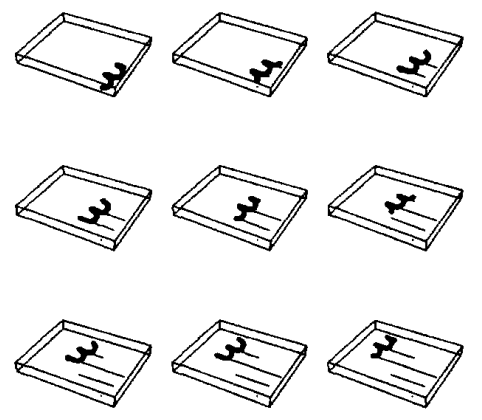

Figure 6: Snapshots of Computer Simulation of Sidewinding Kinematics

robot consisting of a concatenation of 10 Stuart platforms implementing the sidewinding gait.

\section{Turning and Changes in Direction}

While sidewinding is a fast gait, it is not the most flexible gait for turning. Recall that the direction of travel is the sum of a component parallel to the GCTs and one along a vector from the beginning of one AS to the beginning of the next. To turn, at least one of these components must change. Below we outline methods for modifying the direction of travel. None of these methods involves sliding. Because of length restrictions, we omit the analytical details.

\subsection{Turning by Placement}

A change in direction can be effected by placing the head on the next GCT at a different relative position (i.e, by changing $d_{\|}$). Fig. 8 is an overhead view of the backbone curve during such a turning sequence. However, this means of turning may
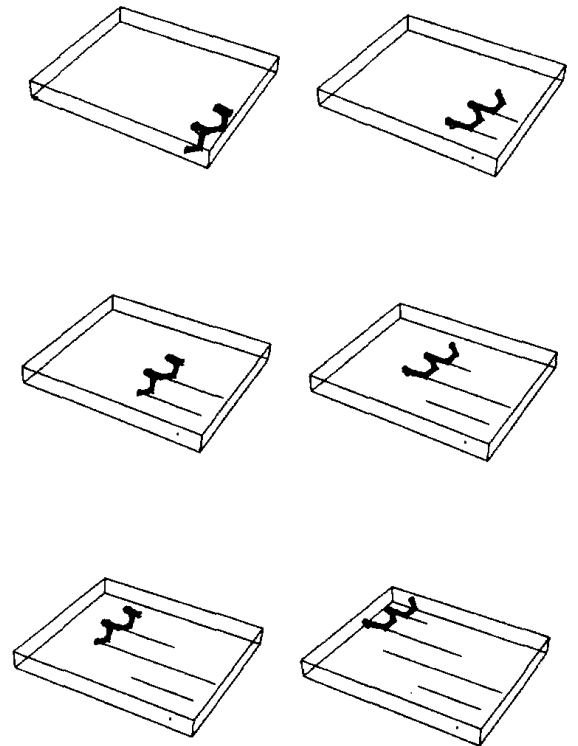

Figure 7: Discrete Morphology Robot implementing the Sidewinding Gait

be unacceptable because the new shape required to move in a given direction may not as efficient or energetically favorable. Temporary deviations from the nominal direction of motion can be implemented by a "jog" (Fig. 9), in which the arch placement is altered only between one pair of adjacent ground contact tracks. This behavior is useful for detouring around obstacles.

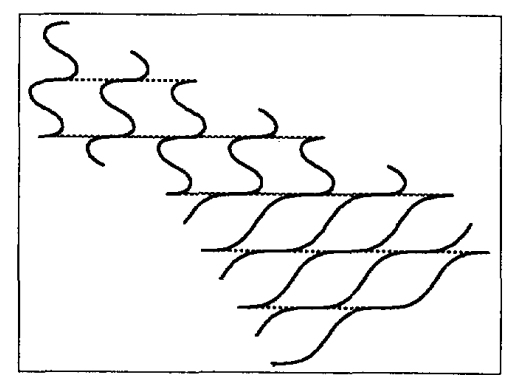

Figure 8: Turning by alteration of head placement

\subsection{Turning via Skew GCTs}

A change in direction can also be implemented by changing two successive GCTs from parallel to skew. This can be done during the arch segment forming phase. The head touches down on the next GCT so that the backbone curve tangent is at an angle to the previous GCT. All subsequent GCTs are parallel to this new skew track. Thus, the sidewinder shape is the same before and after the transition Note that there is a limit of on the 


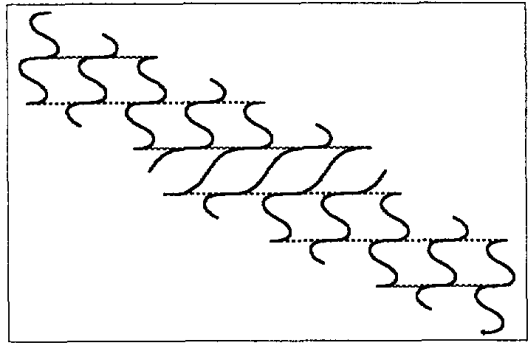

Figure 9: A "jog" in arch placement

If the skew angle is too large, depending on the direction of turning, the snake must intersect itself at some point during the motion, or the distance between the skew ground contact tracks will exceed the reach of the AS in the transition region. Thus, this method is limited to wide turns.

\subsection{Turning by Curvilinear GCTs}

Turning can also be implemented by "bending" the ground contact tracks during the turning sequence. As in the skew method, the GCTs are parallel before and after a turning transition. However, in the transition region, the ground contact lines are curved to smoothly blend the two sets of parallel lines. During the turning process, the snake is laid down along the curved GCTs (Fig 10). Note that turning is most easily accomplished if the length of the GCS is small.

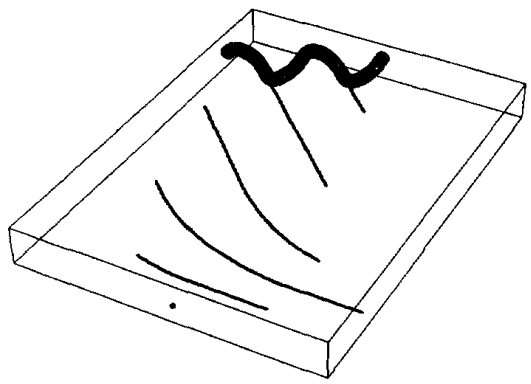

Figure 10: Turning Via Curved Ground Contact Tracks

Note that in this method and the skew method, the arch segment wave shape is not constant. Thus, more complicated arch segment shape functions employing at least three modes are required. Consequently, the modal Jacobian method must be used to compute the arch segment shape which tracks the changing displacement between points on the GCTs in the turning transition region.

\section{Conclusion}

This paper presented an analysis of and a set of algorithms for hyper-redundant robot locomotion which is analogous to snake sidewinding motion. This analysis focused on continuous morphology mechanisms, but we showed by way of example that a discrete mechanical structure can also implement this gait. This gait, which extends our previously developed repetoire of hyper-redundant locomotion gaits, is most useful for fast gross displacement. This paper did not consider sidewinding locomotion over uneven terrain. Sidewinding is really only feasible and advantageous over relatively level ground. [ChB93b] developed algorithms for curvilinear locomotion over uneven terrain. [ChB93b] used the same kinematic framework as this paper, and thus it is possible to smoothly transition from a sidewinding gait to one of the rough terrain curvlinear traveling wave gaits studied in [ChB93b].

Acknowledgements: This work was supported by NSF grant MSS-901779, by NSF PYI award MSS-9157843, and by ONR Young Investigator Award N00014-92-J-1920.

\section{References}

[Ch92] Chirikjian, G.S., "Theory and Applications of Hyper-Redundant Robotic Manipulators," Ph.D thesis, CAL TECH, June, 1992.

[ChB90a] Chirikjian, G.S., Burdick, J.W., "An Obstacle Avoidance Algorithm for Hyper-Redundant Manipulators," Proc. IEEE Int. Conf. on Robotics and Automation, Cincinnati, Ohio, pp. 625-631, 1990.

[ChB91a] Chirikjian, G.S., Burdick, J.W., "Parallel Formulation of the Inverse Kinematics of Modular Hyper-Redundant Manipulators," Proc. IEEE Int. Conf. on Robotics and Automation, Sacramento, California, pp. 708-713, April 1991.

[ChB91b] Chirikjian, G.S., Burdick, J.W., "Kinematics of Hyper-Redundant Locomotion with Applications to Grasping," Proc. IEEE Int. Conf. on Robotics and Automation, Sacramento, California, pp. 720-725, April 1991.

[ChB92a] Chirikjian, G.S., Burdick, J.W., "On the Determination of Kinematically Optimal Hyper-Redundant Manipulator Configurations," Proc. IEEE Int. Conf. on Robotics and Automation, Nice, France, May 10-15, 1992.

[ChB92b] Chirikjian, G.S., Burdick, J.W., "Design, Implementation, and Experiments with a 30 Degree-of-Freedom Hyper-Redundant Robot," Proc. IEEE Int. Conf. on Robotics and Automation, Atlanta, GA, May, 1993.

[ChB92c] Chirikjian, G.S., Burdick, J.W., "A Modal Approach to Hyper-Redundant Manipulator Kinematics," submitted to IEEE Trans. on Robotics and Automation.

[ChB93a] Chirikjian, G.S., Burdick, J.W., "Planar HyperRedundant Robotic Locomotion," submitted to the IEEE Trans. on Robotics and Automation.

[ChB93b] Chirikjian, G.S., Burdick, J.W., "Curvilinear Hyper-Redundant Robotic Locomotion Over Uneven Terrain with Applications to Grasping" submitted to the IEEE Trans. on Robotics and Automation.

[Gray] Sir James Gray, Animal locomotion, Weidenfeld \& Nicolson, London, 1968

[HiMo90] Hirose, S., Morishima, A., "Design and Control of a Mobile Robot with an Articulated Body," Int. J. of Robotics Research, vol. 9., no. 2, pp. 99-114, 1990.

[Secor] S.M. Secor, "Adaptive Strategies of Thermoregulation in Free-Ranging Sidewinders CROTALUS. CERASTES," American Zoologist, 29(4), pp. 9, 1989.

[SJB] S.M Secor, B.C. Jayne, and A.F. Bennett, "Locomotor Performance and Energetic Cost of Sidewinding by the Snake Crotaius-Cerastes," J. Exp. Biology, vol. 163, pp. $1-14,1992$. 